\title{
Didáctica de la Educación Tecnológica: Hacia un modelo explicativo - cultural para el aprendizaje de la tecnología ${ }^{1}$
}

\author{
Didactics of Technology Education: Toward a descriptive-explanatory model for \\ learning technology
}

Dra@Marcela Romero Jeldres

Universidad de Santiago de Chile. Departamento de Educación. marcela.romero@usach.d

Recibido: 26-04-2009

Resumen

El propósito fundamental de este trabajo es caracterizar y promover nuevos modelos de formación inicial y continua de profesores de educación primaria y secundaria, cuyo foco sea el aprendizaje de la tecnología y la promoción de una cultura ciudadana basada en el desarrollo humano diverso, plural e innovativo. Para ello, se presenta un breve panorama histórico del escenario chileno e internacional que precede a la incorporación de la tecnología en el curriculum y su devenir en la enseñanza, luego a la luz del "Proyecto FONIDE No:FIE -2006 FIE_0000218MINEDUC: Innovación y desarrollo en la enseñanza de la Educación Tecnológica: Escenarios para una nueva cultura docente", se propone un modelo didáctico explicativo-cultural para el aprendizaje de la tecnología en los niveles primarios y secundarios, desarrollado sobre la base de los saberes pedagógicos manifiestos en los Planes y Programas de estudio del estado chileno para el subsector de Educación Tecnológica.

\section{Palabras claves}

Didáctica de la Educación Tecnológica, Educación Tecnológica, Formación de profesores, Alfabetización tecnológica.

\section{Abstract}

The primary purpose of this study is to characterize and promote new models of initial and continuing training of teachers in primary and secondary education, whose focus is learning the technology and the promotion of a civic culture based on human development diverse, pluralistic and innovative. To this end, a brief historical overview of the Chilean and intemational scene that precedes the incorporation of technology into the curriculum and their future in teaching, then in light of the "Project FONIDE No: FIE-MINEDUC -2006 FIE_0000218: Innovation and development in the teaching of Technology Education: Scenarios for a new educational culture ", an explicativecultural teaching model for teaching technology in primary and secondary levels, developed on the basis of knowledge is manifest in pedagogical Plans and Programs of study for the Chilean state subsector Technological Education.

\section{Keywords}

Teaching of Technology Education, Technology Education, Teacher training, technology literacy.

\section{Introducción}

\footnotetext{
${ }^{1}$ Este artículo forma parte del capítulo teórico de la tesis doctoral "Educación Tecnológica en el curriculum chileno: Implementación, prácticas docentes, sentido y cambio en el hacer", de esta autora. 
A igual que en Chile, el común denominador con el resto de los países que incorporan la Educación tecnológica, "tiene una especie de antecedente histórico- generalmente en educación de manualidades, los cambios hacia la Educación Tecnológica actual son tan fundamentales que, fácilmente, uno podría justificar que se habla de un nuevo tema" De Vries (2001:19), por lo tanto, la enseñanza de la Educación tecnológica "comienza a significar una preocupación prioritaria en numerosos países... Podemos constatar que ésta no se trata de la misma manera, pudiendo variar de un país a otro..en una perspectiva internacional, sobresale como parte de la cultura general" (Ginestié, 2001:31).

El escenario del siglo XXI enfrentado al fenómeno económico y financiero, llamado globalización, nos obliga a convivir con diversidad de procesos cient́ficos y tecnológicos, enlazados planetariamente por las tecnologías de la información y la comunicación, (en adelante Tics), y donde "La enseñanza científica no puede dar cuenta por sí sola de los hechos tecnológicos y, por otro lado, la educación técnico manual circunscrita al espacio doméstico no permite dar un sentido o significado a la tecnología". La Porte (2001; 91).

Es de este modo que se justifica la incorporación de este subsector en los diferentes curriculum, La Porte (2001; 91) señala que "la teoría para la adopción de la Educación Tecnológica generalmente está basada en la noción de que vivimos en un mundo tecnológico. Todo ciudadano necesita saber de tecnología, de manera que pueda utilizarla apropiadamente y tomar decisiones informadas sobre ella, en su beneficio y en el de la sociedad".

Aguayo, et al (1998; 4:8) sostiene que las principales influencias de todas las incorporaciones, de los países que la han induido, están primeramente asentadas desde la educación técnica, planteada por el filósofo Whitehead, luego en los trabajos del grupo Villa Falconieri Frascati (Italia), que la introduce fundamental en la enseñanza secundaria, como un instrumento operativo y de comunicación fundamental de la sociedad modema; la tercera influencia se organiza desde la reunión de Sevres del Consejo de Europa a finales de 1965, que hacen consideraciones a los contenidos, metodologías didácticas y orientaciones de la tecnología; la cuarta influencia, el informe Porter del Reino Unido en 1967, refuerza el informe Crowther de 1959 extendiendo la enseñanzas a los centros de educación general, en tres niveles: de 11 a 13 años; de 14 a 16 años; de 16 a 18 años.; la quinta influencia es el Simposio de Nottingham el año 1972, sus condusiones hicieron dificil diferenciar entre la cultura tecnológica y la tecnología necesaria en la formación profesional.; la sexta se refiere a las experiencias en Sevres y sintetizadas por Capelle, quien sostiene que la tecnología es un lenguaje, un medio cultural, y una disciplina en construcción.; la séptima se refiere a las artes industriales introducidas por los Estados Unidos, en la escuela elemental obligatoriamente con un significado de tipo experimental, y en la Enseñanza Secundaria opcional; finalmente las jomadas intemacionales de Educación Tecnológica celebradas en Barcelona en 1997, hacen hincapié en la enseñanza del diseño y la tecnología, en los valores y juicios como el motor del diseño y la tecnología sobre las carencias, intereses y preferencias de las personas.

En este sentido, las sociedades modernas vienen incorporado modificaciones en sus curnículos que permitan reemplazar las técnicas manuales por "nuevos campos de saberes, de actividades, de campos de dominio de referencia y posturas" (Ginestié, 1998 (a)) Hömer(1987), no sin largas discusiones y enfoques intemacionales. "propone una descripción de curríallos que fue complementado por la fundación Pupils Attitude Towards Technology a nivel mundial" [Raat, 1985, De Klerk-Wolter,1987, De Vries,1989)]. A respecto, De Vries (1995) "propone una metodología según diversos enfoques para la educación tecnológica en la escuela primaria", otros enfoques son desarrollados por WOCATE (World Council Assodations for Technology Education), ellos sugieren "una extensión (...) desde la primaria hasta las formaciones profesionales universitarias" (Blandow, Dyrenfurth, 1992).Con ello muestran que la idea de continuidad entre educación inicial y formación profesional está largamente presente y explícitamente aceptada. (Ginestié, 2001; 32) Por lo tanto, y sobre la base de la experiencia de los países que la han incorporado, plantea que la posición de 
adoptar la educación tecnológica, no está en discusión, "\% discutible todavía es definir cuál es la mejor forma de Educación Tecnológica para cada país"La Porte (2001:91)

Ahora bien, la incorporación curricular de la Educación Tecnológica, no es igual en todos los países que la han adoptado. Estados Unidos, por ejemplo, ha realizado una transición desde las artes industriales, hacia los propósitos que una educación general puede dar, desarrollado a través del "Proyecto Tecnología para Todos los Estadounidenses" (TFAAP), en un esfuerzo curricular que induye preescolar hasta secundaria. (K-12). El documento final entregado en el año 2000 se tituló "Estándares para la Alfabetización Tecnológica" (Dugger, 2000), ayya finalidad es proporcionar orientaciones para la Educación Tecnológica, considerada como proceso y contenido (La Porte (2001; 93), del mismo modo, se conceptualizan dos definiciones referidas a la tecnología, la primera la refiere como: "Innovación humana en la acción que involucra la generación de conocimientos $y$ procesos para desarrollar sistemas que solucionan problemas $y$ amplían las capacidades humanas" y la segunda como: "Innovación, cambio, o modificación del medio ambiente natural para percibir y querer satisfacer las necesidades humanas". En el mismo texto se define a la Educación Tecnológica como: "El estudio de la tecnología, que ofrece una oportunidad para que los estudiantes aprendan acerca de los procesos y el conocimiento relacionados con la tecnología necesarios para resolver los problemas y ampliar la capacidades humanas"en Standards for Technological Literacy: Content for the Study of Technology (c) ITEA, 2000/2002/2007. (pág.242).Igualmente, se explicita que la tecnología es un producto y un proceso que implica tanto a la ciencia y a la ingeniería, lo que significa aceptar que se necesita mucho más que la ciencia aplicada para crear o mejorar la tecnología, aun cuando muchas personas siguen atribuyéndole los avances tecnológicos exdusivamente a la ciencia, confundiendo y despreciando la combinación de papel de la ciencia, la ingenieńa y la tecnología en la configuración de la vida modema.

La National Academy of Engineering en adelante NAE, sostiene que la Educación Tecnológica en EEUU, es un tema académico nuevo que está implementada, sólo en 14 estados generalmente asociadas con la preparación técnica., sin embargo, ellos son consientes del contraste que presentan en relación con otros países como República Checa, Francia, Italia, Japón, Países Bajos, Taiwán y el Reino Unido, donde la educación tecnológica se incorpora en la escuela intermedia y/o secundaria. Sumado a lo anterior, una encuesta realizada por la Asociación Internacional de Educación Tecnológica, en adelante ITEA, sugiere que la escasez de profesores de la enseñanza de la tecnología es mayor que el déficit general en la fuerza laboral docente, añadiendo que las escuelas de educación tampoco preparan maestros capacitados para el desarrollo de la alfabetización tecnológica, esto implica que adolecen de una integración del contenido con otras materias, como ciencias, matemáticas, estudios sociales, inglés, y arte, sumado a la escasez de estudios en la disciplina reflejadas en las pruebas estandarizadas.

En el caso de Taíwán, los cambios tecnológicos juegan un factor dave en la vida social y económica, dado que tanto el conocimiento, las habilidades y las actitudes sobre la tecnología influyen en el desarrollo nacional (Zhang, 1999 en Fang, Teng, and Chih-chia: 2007; 7).Según el Informe de 2005 del International Institute for Management Development (IMD), Taiwán ocupa el segundo lugar en el mundo en tecnología competitiva (Lee; 2004), "Esta dasificación hace necesaria la incorporación de la Educación Tecnológica si se quiere seguir siendo competítivos para una variedad de razones, facilitar la adaptación social, la mejora del desarrollo económico, induyendo el desarrollo de talentos tecnológicos y la solución de problemas tecnológicos. El mismo autor plantea que los problemas críticos que enfrentan en Taiwán, son similares a los de otros países sintetizados en los siguientes apartados:

a) El público de Taiuán confunde a menudo la tecnología con la técnica, o como ciencia aplicada, la mayoría de las personas la ven como artefactos, eléctricos o productos informáticos.

b) Los educadores de educación tecnológica han sostenido que el auerpo de conocimientos de la disciplina es la praxis, una combinación de mente $y$ manos, sin embargo los exámenes 
académicos nacionales para la enseñanza superior no han induido estudios, manteniéndola como una asignatura subordinada.

c) Apenas se apoyan programas de educación tecnológica, tales como el financiamiento de investigaciones y desamollo de proyectos.

d) Las escuelas primarias carecen de profesores competentes, y las escuelas seaundarias hacen caso omiso debido a la escasez de docentes.

e) El sistema de prestación de servicios de instucción de la educación tecnológica presenta una falta de coordinación.

Frente a países con mayor trayectoria de implementación, se auentan los casos de Reino Unido y Francia, en el primero, la Educación Tecnológica presenta una historia en el curriculum que " arranca con las enseñanzas del arte y artesanía que se hacía a principios de siglo, pasa por la enseñanza de las habilidades manuales para los alumnos menos dotados durante la década de los 40, incorporando estudios técnicos y de economía doméstica para los alumnos medios, en la década de los 50 y 60, establece la enseñanza general de CDT (Craft, Design and Technology) y de la economía doméstica a partir de los 70, aulmina con la implantación del National Curniculum de 1990, que establece la Technology como asignatura obligatoria en primaria y secundaria para todos los alumnos"Font(1996;20).

Para Cruz, A. y R. McCormick (1986), el desarrollo de la educación tecnológica en el Reino Unido es un ejemplo de un enfoque fructífero para ayudar a los estudiantes en la comprensión. El diseño es una parte esencial de la tecnología y el Reino Unido rinde una gran atención a ello para aprender a resolver problemas tecnológicos "Los estudiantes suelen ofrecer soluciones tecnológicas a. problemas que son el fundamento para las patentes de industrias. Por esa razón, la tecnologíaen el Reino Unido- ciertamente merece atención".

Para el caso de Francia, la educación tecnológica se incorpora como una iniciación cientúfica y tecnológica en la Educación Básica, seguida de Tecnología en la Educación Media, en la franja de 12-16 impartida en los college desde 1984.Curricularmente, la tecnología como asignatura, se focaliza en una formación cultural y no en una formación profesional. Metodológicamente se busca el equilibrio entre módulos de contenidos y el trabajo de proyectos, focalizando, en una primera etapa, en el análisis de objetos tecnológicos y la resolución de problemas y hacia el final del cido, en la realización de proyectos que puedan realizarse en los centros focalizados en: mecánicaautomática, electricidad-electrónica con informática industrial y economía-gestión, induyendo informática y conocimiento del mundo laboral.

Respecto de los docentes franceses, la inclusión de este curriculum, hizo necesaria la reconversión del profesorado, "Se tomó el profesorado que obligatoriamente se tenía que reconvertir y el que quiso hacer voluntariamente la nueva materia y se le formó fuera del centro durante un aurso académico de 900 horas.(...) el contingente de enseñantes que se twro que recidar obligatoriamente estaba formado por profesorado de las antiguas enseñanzas del hogar, de las antiguas actividades técnicas y manuales, por maestros sobrantes de primaria, por instructores de formación profesional'. De este modo, para ejercer docencia, los profesores de esta especialidad deben poseer un tútulo profesional más un curso de capacitación pedagógica (CAPET) que induye la especialización en tecnología para la enseñanza obligatoria, "En cada distrito universitario hay un equipo de asesores e inspectores de tecnología que se ocupan exdusivamente de los aspecto aurniaulares, de las prescripciones de los equipos de las aulas-taller, de los centros de recursos y del recidaje del profesorado"Font (1996; 19).

En relación con la implementación de la Educación Tecnológica en el curriculum Finlandés, Aki Rasinen (2003; 42), analiza el plan de estudios de seis países en que los programas de educación teconológica se han desarrollado rápidamente en los últimos diez años, llevando a cabo desarrollo de materiales de aprendizaje, investigación y programas experimentales, a saber: Alemania, Australia, 
Inglaterra, Francia, Países Bajos, Suecia y los Estados Unidos. Los resultados globales de este análisis de similitud en los documentos curriculares, dan cuenta que estos seis países se encuentran en diferentes fases de desarrollo de sus programas de educación tecnológica, sin embargo el punto de partida es la planificación del currículo, el proceso de planificación, aun cuando la estructura del currículo difiere de un país a otro. En relación a los planes de estudios, estos plantean características similares en sus objetivos curriculares, métodos y contenidos, siendo la alfabetización tecnológica un objetivo universal.

Respecto de los objetivos de estudio, el foco está puesto en la comprensión del papel de la ciencia y la tecnología en la sociedad, el equilibrio entre la tecnología y el medio ambiente, el desarrollo de la alfabetización tecnológica y el desarrollo de capacidades como la planificación, elaboración, evaluación, social y moral-ética de pensamiento, la innovación, el conocimiento, la flexibilidad y el espíritu empresarial. A cerca de los métodos, ellos se centran en las experiencias de los estudiantes que promueven planificar, analizar, inventar, innovar, hacer, y evaluar. En relación con los contenidos, estos abordan los sistemas y estructuras de la tecnología, las profesiones, la industria, las prácticas de seguridad, la ergonomía, el diseño, las técnicas de construcción, las prácticas de evaluación, el papel y la historia del desarrollo tecnológico, estrategias de resolución de problemas, y evaluar y valorar la relación entre la sociedad y la naturaleza.

En el caso chileno, su historia también sigue el mismo origen de las manualidades, desde la época republicana "todo el sistema de aprendizajes de artesanías, fuente de riquezas de los pueblos europeos y base de su industria, faltaba por completo en nuestra educación'( Labarca, 1939;73), por lo tanto, el surgimiento, en Chile de la Escuela de Artes y Oficios en 1846, viene a suplir la carencia de conocimientos y prácticas en tomo al estudio de la química y la mecánica en los ramos de manufacturas e industrias agnícolas, como una forma de superar los problemas que impedían a Chile entrar en la ruta del progreso En ese camino, la Ley de Enseñanza de 1860, estableció materias de estudio elementales para hombres y mujeres en tomo al dibujo lineal y la economía doméstica, costura bordado y labores de aguja.

En 1881 se incorpora la asignatura de trabajo manuales a todo el sistema educacional, cuando el gobiemo de la época, contrata a profesores suecos para introducir el método educativo s/öyd: sistema de preparación manual basado en la enseñanza de trabajos con madera. En 1888 el Consejo de Enseñanza Agrícola e Industrial, propone la creación de una escuela de artes y oficio para mujeres, abriendo un espacio de enseñanza práctica cuya especialidad era marcadamente diferenciada por lo que se estipulaba como "femenino" y referido mayormente a trabajos manuales tales como: comercial; modistas; lencería y costura corriente, bordados, guantería, cartonaje y marroquinería, cocinería, lavado y planchado, también dibujo. La edad de ingreso se estipula en los doce años aumplidos y con los conocimientos previos otorgados por la escuela primaria, siendo obligatorias las materias de dibujo. En el caso de los hombres, la edad mínima de ingreso era de 14 años con un máximo de 17, se les solicitaba una prueba de conocimientos en varias materias tales como: Castellano, matemáticas (aritmética y geometría) e historia, los tres primeros años podían optar a un título de "Operario" fundidor, herrero, calderero, hojalatero, carpintero, modelista, mecánico o electricista y al cabo de cinco años podían obtener el título de Técnico mecánico, electricista o químico. Reglamento para la Escuela de Artes i Oficios (1864:22).

Con la reforma de 1907 se induye la enseñanza del trabajo manual en todos los niveles, de la escuela primaria, separados por género y dando énfasis a los trabajos en madera, tejidos y alimentación. La reforma de 1966 mantiene la línea de trabajo, pero incorpora dos asignaturas: Educación Técnico Manual, para Educación General Básica y Técnicas Especiales para la Educación Media. Esta asignatura tiene como objetivo que los estudiantes pudiesen adquirir conocimientos y destrezas manuales para fabricar objetos utilitarios. De esa manera, el foco del contenido estaba puesto en aprender técnicas y procedimientos para manipular herramientas y transformar materiales. Estas transformaciones se realizaban poniendo el foco en el desarrollo individual de los 
estudiantes de la motricidad fina y gruesa, la precisión, rapidez y la fuerza. Del mismo modo, mantiene la diferenciación histórica del trabajo manual por género desde 1864. Las formas de evaluar se organizaban en función del producto terminado y de lo utilitario del objeto fabricado.

Ciento treinta y dos años después, el Decreto №40 de 1996, elimina "Técnico-manual", y crea, con el mismo estatuto jurídico que la enseñanza de las matemáticas, la lengua y las ciencias, un nuevo Subsector de aprendizaje llamado Tecnología y dentro de éste, el Subsector de Educación Tecnológica, obligatoria para los estudiantes desde primero básico a segundo año medio $\left(1^{\circ}\right.$ a $10^{\circ}$ grado) y opcional para III y IV año de Educación Media, (11y $12^{\circ}$ grado). El fin ministerial es que los estudiantes adquieran conocimientos tecnológicos para ser usuarios y consumidores aíticos e informados de tecnología. Pretende desarrollar en los estudiantes, la capacidad de comprender la teconología como cultura, distinguiendo la intencionalidad humana detrás de un producto tecnológico o un servicio y cómo éstos, intervienen el mundo social y medio ambiental. La incorporación de este subsector de aprendizaje, obliga a definir algunas conceptualizaciones de tecnología, en tanto ésta se configura como su objeto de estudio.

Una rápida y escueta mirada, hoy, a trece años de su puesta en marchar en Chile, da cuenta que esta implementación, corre por suertes similares respecto de otros países que la integran en su instalación como conocimiento tecnológico en tanto saberes conceptuales, metodológicos, actitudinales y axiológicos y decodificados sobre una base común de saberes estructurados progresivamente. Sumado a lo anterior, las investigaciones que dan cuenta de esta implementación, no son más auspiciosas, evidenciando una dificultad en consensuar el conocimiento que se ha de aprender, el cómo se ha de enseñar, qué recursos pedagógicos se deben movilizar, quienes deberán ayudar a esa formación, dónde y cómo se han de medir -dado que ellos que no son requeridos en pruebas intermacionales tales como PISA;TIMSS y PIRLS, ni nacionales como SIMCE en (Abraham,2000; Romero,2006; Romero et al, 2007), de modo tal, que sumado al desconocimiento que tiene la sociedad civil, resultaría muy probable que si "mañana" fuese eliminada del curriculum, la opinión pública y los medios de comunicación poco dirían de esta asignatura, que de ser una ramo pendiente, muchas veces pasa a ser una asignatura olvidada.

\section{Hacia la construcción de la didáctica de la Educación tecnológica}

\section{Conceptualizaciones para la didáctica}

Como la tecnología no es una materia académica que se transmita como un conocimiento homogéneo invariable, hace que integremos la actividad humana como un elemento inseparable de la historia, del lenguaje, de la cultura y de los efectos que la tecnología genera en la sociedad. Esta mirada nos obliga, a no sólo poner el acento en transmitir aquellos aspectos concretos y humanos en que la tecnología nos ha afectado o beneficiado, dándonos una identidad como sociedad, sino también, en propiciar el aprendizaje de la tecnología, situado desde la cultura tecnológica local o global, pero con una actitud crítica frente a ella. De este modo, "al darle a la tecnología la condición de conocimiento humano, es posible reconocer en ella, objetivos, contenidos, procedimientos, actitudes y haceres, y como tal, reconocerle un aporte identitario en la cultural planetaria, vista así, no se la puede desvincular del culrículo, como tampoco se la puede asociar sólo con la formación profesional, con la adquisición de destrezas manuales, con prácticas experimentales 0 con la historia de la ciencia y de la tecnología'. (Romero et al 2007; 21).

Por tanto, para darle un sustento epistemológico al aprendizaje de la Tecnología, se hace vital incorporar en la Didáctica de la especialidad, elementos teóricos que dicen relación con la incorporación de aproximaciones filosóficas de la tecnología, dado que ellas permiten "interpretar y/o construir el mundo artificial desde concepciones que ven al hombre como un ser dominante que realiza acciones tecnológicas centradas en él y sus necesidades o bien desde conceptualizaciones 
que auestionan la tecnología y le interesa conocer la real relación que hay entre ésta, lo humano, y el manejo tecnológico' Mitcham (1989; 21-49).

Sumado a lo anterior, se hace necesario dilucidar si la tecnología tiene un estatus cognoscitivo de ciencia o si se le seguirá considerando como una ciencia prolongada, inhibiendo el desarrollo de la filosofía de la tecnología, y donde su relación con las ideas no es tan explícita, como la relación entre ciencia e ideas, no obstante, Mitcham (1989; 100) señala que "existen ideas distintamente tecnológicas como se revela en las ciencias tecnológicas. El concepto de máquina (...), las ideas de conmutador, invención, eficiencia, optimización, la teoría aerodinámica, la cinemática y la cibernética, las teorías de autómatas, de la información, de los sistemas lineales, del control, etc, son esencialmente tecnológicas", por otro lado y dado el carácter práctico de las ideas tecnológicas, no es posible interrogar a las teońa tecnológicas como verdaderas o falsas, sino si ellas funcionan, o si son buenas o útiles, "cuando se plantean dudas sobre ello o sobre la talla moral de las acciones tecnológicas y sus resultados así como de las ideas en las aules ellas se basan, entonces, se comienzan a desarrollar ideas en vez de simples teorías de la tecnología" Mitcham (1989; 101), estas ideas y cuestionamientos, señala el autor, van desde lo conceptual hasta lo metafísico.

De este contexto anterior, se nutre la Didáctica de la Educación Tecnológica, recogiendo además las nuevas problemáticas que este siglo demanda en materia de tecnología. Ellas nos llevan a remirar el acto didáctico, desde los intereses de los niños, niñas y jóvenes que deben alfabetizarse en estas materias para ser ciudadanos, tomadores de decisiones tecnológicas informadas y éticos. Encarnar estas problemáticas, es un cuestionamiento que la didáctica de ésta especialidad no puede eludir. ¿Cómo ayudamos para asumir estos nuevos desafíos desde la escuela? ¿Qué metodologías seguimos para implementar estos procesos?

Ahora bien, al reconocerle a la tecnología su estatuto cognoscitivo, es posible decir que así como el método científico es a la ciencia, el método de proyecto es a la tecnología, entonces se hace necesario enriquecer este proceso de búsqueda, teniendo presente, la incorporación de estrategias abiertas, flexibles, movidas por ideas-fuerza, que caractericen las situaciones complejas que enfatiza la tecnología, posible de ser abordadas desde un pensamiento complejo y donde la colaboración en la resolución de problemas no tiene una mirada centrada sólo en la eficiencia y eficacia de la gestión de recursos para producir una solución, sino en la búsqueda de las cuestiones éticas de la tecológica modema y la responsabilidad social que tiene la decisión colaborativa, hacia allá apunta el método de proyectos y su aplicación al campo de la educación escolar.

De este modo, estos focos y sus sustentos históricos-filosóficos, sin duda relevan la construcción de una didáctica de la disciplina y le dan, por añadidura, una responsabilidad social. Eso implica que las opciones que se tomen para intencionar el aprendizaje-enseñanza, desde alguno de estos enfoques (ingenieril o humanista), señalarán un lineamiento y una mirada del mundo, para comprender e interpretar el impacto social, cultural y medio ambiental que han tenido los últimos desarrollos científico- tecnológicos. En ese escenario es, que surge a modo de metodologías, el Modelo explicativo-cultural para la enseñanza de la educación tecnológica explicitado en el marco del "Proyecto FONIDE No: FIE -2006 FIE_0000218-MINEDUC: Innovación y desarrollo en la enseñanza de la Educación Tecnológica: Escenarios para una nueva cultura docente", (Romero et al 2007)

\section{Modelo explicativo-cultural para la enseñanza de la educación tecnológica}

Sumada a las metas y concepciones de las ciencias tecnológicas mencionados anteriormente, en este apartado, y a modo de síntesis de la investigación, hablaremos de "modelo", para referimos a la manera de enlazar los elementos abstractos de la tecnología con lo concreto y funcional de ella, hablaremos de "explicativo" para referiremos a las representaciones conceptuales, esquemáticas y/o simbólicas de mediación entre la experiencia de investigación llevada a cabo en el campo de 
aplicación escolar y la interpretación final de esa realidad vivenciada; del mismo modo hablaremos de "cultural" para dar cuenta de la secuencia que fue tomando cada método propuesto desde los lenguajes de los docentes que lo aplicaron y las modificaciones que solicitaron los estudiantes que lo vivieron. Es importante mencionar que dado el carácter cualitativo de la investigación de base, estas elaboraciones son propuestas a implementar y validar.

\section{Veamos:}

Conceptualizamos la Educación Tecnológica como "Un proceso educativo que permite a los estudiantes, el desarrollo y la aplicación de competencias para comprender el mundo artificial y analizar sus impactos sobre el medio ambiente, la vida humana y los cambios sociales y para la proposición y elaboración de soluciones tecnológicas como respuestas creativas a necesidades detectadas. Sostenemos que la tecnología es el objeto de estudio de la Educación Tecnológica y por tanto nos sumamos a entenderla como "Una actividad social centrada en el saber hacer que mediante el uso racional, organizado, planificado y creativo del conjunto de los recursos materiales y de la información, propios de un grupo humano, en cierta época, brinda respuestas a las necesidades y 10 demandas sociales en lo que respecta a la producción, distribución y uso de los bienes, procesos y serviaios." CBCEGB. (1995).Conceptualizamos la Didáctica de la Educación Tecnológica como "un área cognoscitiva que estudia los procesos de construcción del conocimiento tecnológico del modo más eficiente bajo todos sus determinantes y niveles educativos, así como todas las tareas derivadas de la práctica docente de la construcción del conocimiento tecnológica". Junyent $(1997,2001)^{2}$

De esta manera, se entiende que para lograr los OFV, OFT y CMO del marco curricular, propuestos por el MINEDUC en el Subsector, la Didáctica de la Educación Tecnológica debe situarse alineada curricularmente con esta propuesta mínima ministerial, es así como se han establecido una serie de aspectos propios del subsector y que fueron motivados por otras experiencias en la enseñanza y aprendizaje de la tecnología, iniciando así una apropiación auténtica en la realidad escolar chilena a saber: El modelo didáctico

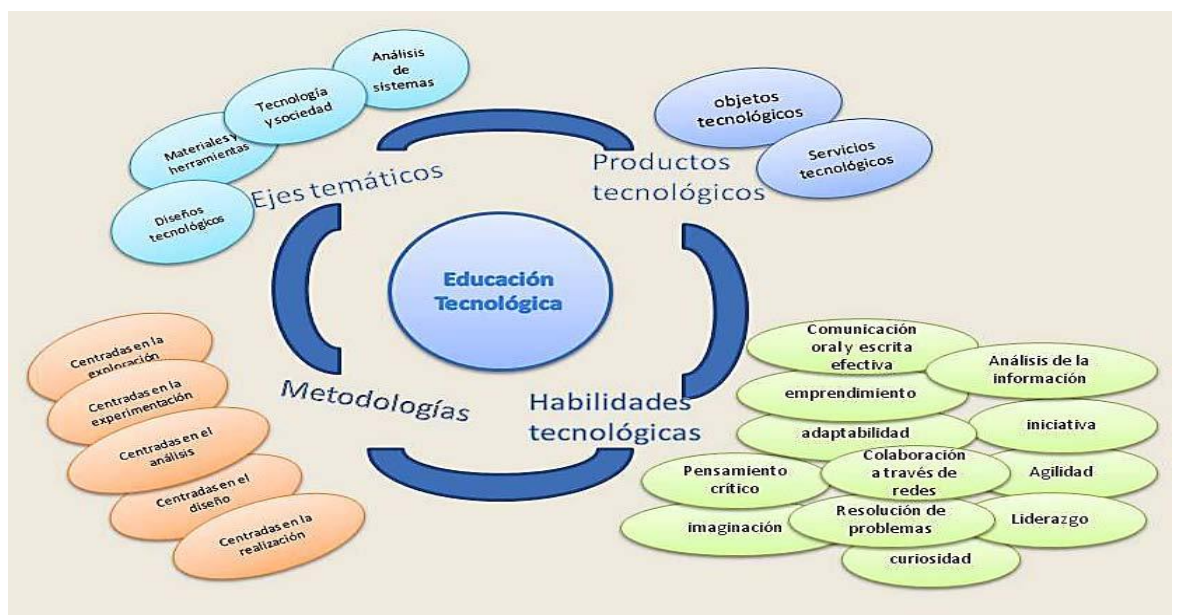

Fig. 1-Modelo didáctico para la enseñanza de la educación tecnológica.

Para desarrollar el trabajo en el subsector, se propone un modelo didáctico que permite establecer estrategias de aprendizaje-enseñanza, apoyados en una estructura didáctica con la que se podrá abordar los contenidos de Educación Tecnológica de una forma progresiva y ordenada en cada sesión de dases. Este proceder didáctico facilitará la planificación de actividades y podrá

\footnotetext{
${ }^{2}$ Estas conceptualizaciones se elaboraron en el Equipo de Educación Tecnológica de la Facultad de Educación de la Pontificia Universidad Católica de Chile, dirigido por la Dra.Junyent para apoyar la implementación curricular del decreto $\mathrm{N} /{ }^{\circ} 40$ 
adaptarse a los diversos tipos de desafios a los que queremos enfrentar a nuestros estudiantes. La secuencia formativa se organiza en tormo a cinco grandes directrices (ejes temáticos, productos tecnológicos, metodologías y habilidades tecnológicas) que se ven iluminados por la lógica de la metodología de proyectos en su base y el sustrato epistemológico que otorga la filosofía de la tecnología.

Ahora bien, para facilitar el tránsito hacia la metodología de proyectos, entendida como la metodología propia de la tecnología, hemos conceptualizamos el proyecto tecnológico como "el trabajo educativo desarrollado por niños, niñas y jóvenes, en dos, cuatro o más semanas de duración con gran particjpación de estos, en su planteamiento, diseño, realización y su posterior evaluación, propiciando la indagación infantil y juvenil que permita evaluar procesos, productos $y$ servicios tecnológicos, con el fin de obtener resultados que les permitan encontrar soluciones creativas tanto para inventar o innovar elementos del entorno tecnológico donde se desenvuelve." (Romero et al, 2007.)Estos proyectos, en un comienzo se organizan desde problemáticas cenradas conducidas para potenciar las habilidades necesarias respecto de la investigación, diseño, construcción y comunicación, para luego abrirse a una participación acompañada, que se sitúa en un ámbito tecnológico y que les enfrenta a plantear y resolver sus propias problemáticas.

Finalmente y para facilitar la apropiación del conocimiento tecológico y el desarrollo y fortalecimiento de habilidades tecnológicas, se han configurado las siguientes metodologías que serán seleccionadas por el docente según los desafíos de aprendizaje que demanden los estudiantes, a saber:

a) Metodología centrada en la experimentación: Permite promover la vinculación científica de la tecnología, modelar técnicas y procedimientos nuevos o ingeniosos con el fin de que los estudiantes, puedan replicar ciertos pasos, sentirse seguros al manipular materiales y herramientas y luego aplicar y transferir estos procedimientos, y conocimientos en la construcción de un objeto que solucione un problema planteado.

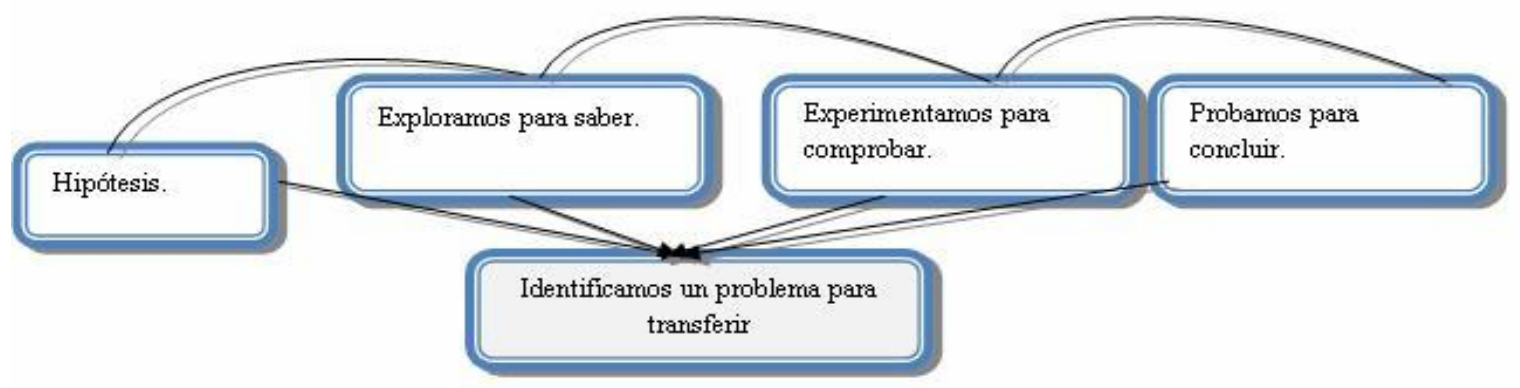

Fig. 2 Metodología centrada en la experimentación.

b) Metodología centrada en la exploración. Se entiende como un examen colectivo, planificado, y organizado de un objeto, sistema, proceso, producto o servicio, perteneciente al contexto próximo del estudiante, con el fin de promover los aprendizajes previstos, sobre el medio ambiente. 


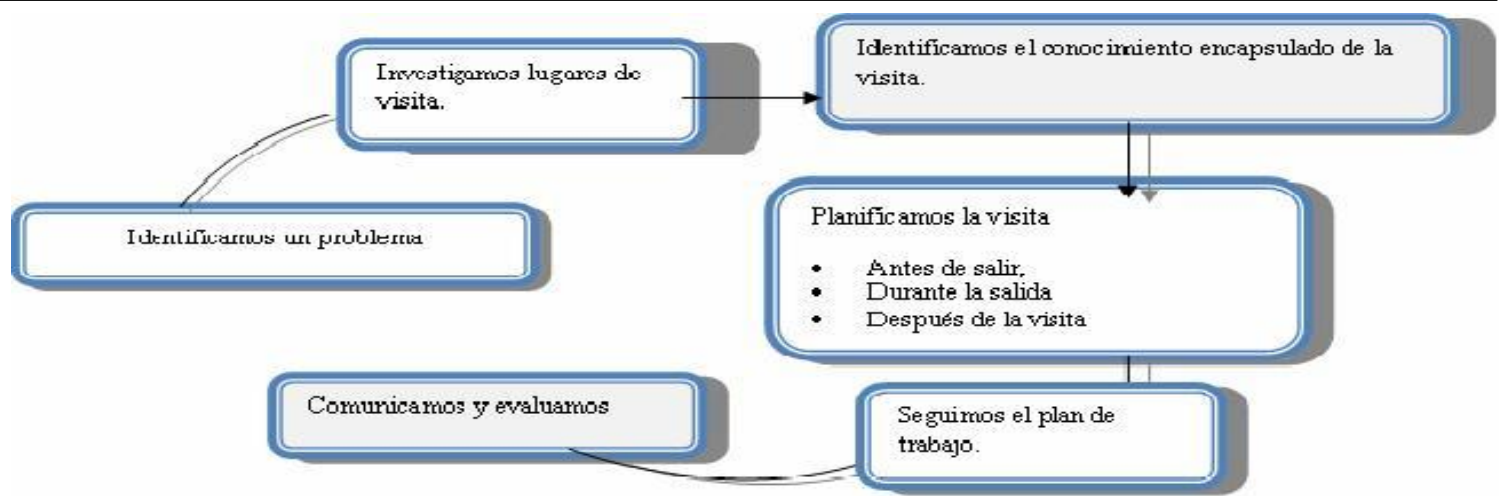

Fig. 3 Metodología centrada en la exploración.

c) Metodología centrada en el análisis: Busca estudiar o examinar productos tecnológicos y/o servicios y para emitir opiniones fundamentadas. Se apoya en el método de análisis y contempla la revisión bibliográfica de documentos para obtener información, que permita tener una visión más acabada del análisis.

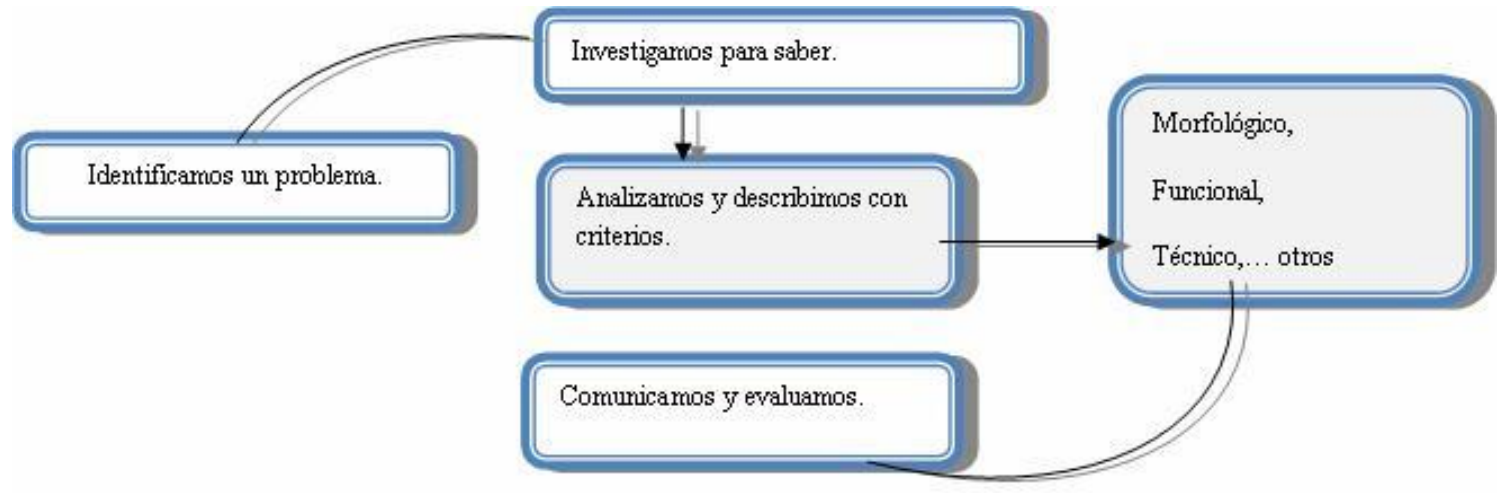

Fig. 4 Metodología centrada en el análisis.

d) Metodología centrada en el diseño: Se entiende así auando se enfatiza la invención y creación de objetos y servicios que mejoran la realidad, de esta manera, el diseño adquiere el carácter de configuración o representación gráfica y conceptual.

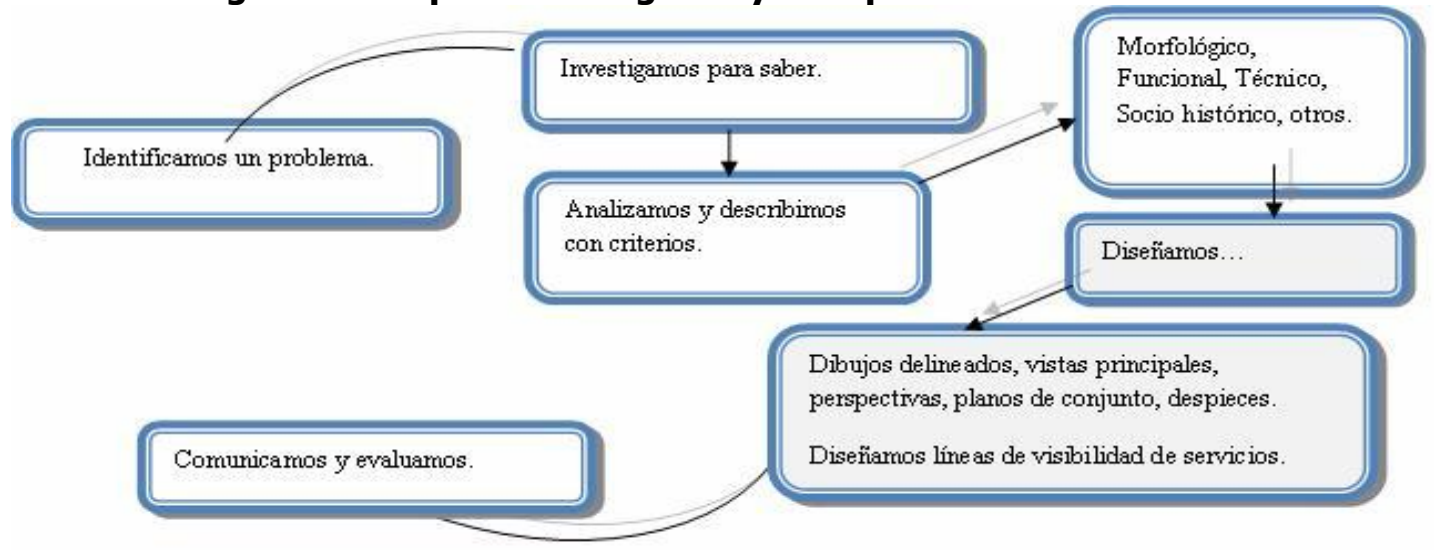

Fig. 5 Metodología centrada en el diseño. 
e) Metodología centrada en la realización: Se denomina así a la tarea que está centrada en la elaboración de un producto tecnológico sea éste un objeto o un servicio. Se apoya fuertemente en todas las fases de la metodología de proyectos y busca familiarizar al estudiante con los procesos productivos, sus fases y la responsabilidad social y medioambiental de las decisiones industriales.

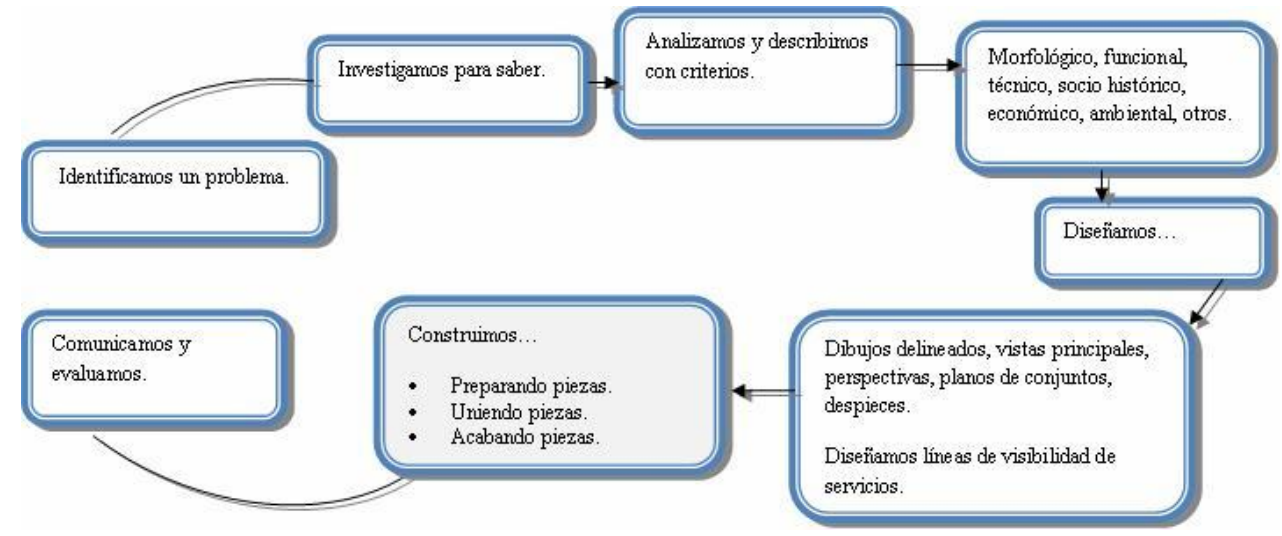

Fig. 6 Metodología centrada en la realización.

Es importante mencionar que este modelo surge del campo de investigación acotado a 10 docentes en activo de educación tecnológica, con diferentes realidades de vulnerabilidad social, en distintas zonas de Santiago de Chile. Con una gran disposición, implementaron y evaluaron en sala, la metodología centrada en la experimentación más la metodología de proyectos en sus fases tradicionales a 850 estudiantes cautivos que pusieron a prueba cada acción docente dado que no siempre contaban con los reaursos materiales, tecnológicos y aulturales que suponía la investigación. Los resultados y los informes preliminares de esta experiencia en particular pueden ser vista en http://w3app.mineduc.d/DedPublico/que es el fonide, del mismo modo este diseño no hubiese tenido el éxito esperado si no hubiese estado respaldado por una web solidaria: unw.tecnociencia.d,también desarrollada en este proyecto y que permite, libremente tomar el material multimedia elaborado y adaptarlo a la realidad escolar que se requiera.

\section{Reflexiones finales}

Chile, no es distinto a otras sociedades democráticas y plurales, que han considerado importante la tecnología como conocimiento de base de los estudiantes, por esa razón, se le ha dado un estatuto jurídico en el marco curricular. Estos nuevos saberes revelan no sólo transformaciones industriales, urbanas y sociales, también evidencian que la falta de desarrollo tecnológico, en el desarrollo productivo de las naciones, nos rotula como naciones con más o menos "grado de cobertura tecnológica" y esto porque la tecnología hoy se anexa no sólo a la ciencia, sino a la economía y la producción industrial, pudiendo hipotecar el crecimiento económico de las naciones ya que ellas determinan no sólo la concepción de la producción industrial, sino también las pautas de gestionamiento, de este modo, para naciones como las nuestras, es imperativo potenciar el desarrollo científico y técnico, no obstante, la noción de progreso prometido por esta vía, no puede desvincularse de la problemáticas éticas ni menos dejarse en manos de sólo las comunidades que producen estos desarrollos o de los cuerpos administrativos y legislativos, debemos avanzar en la alfabetización de un público democrático educado en ciencia y teconología.

En esta idea es que se han realizado esfuerzos por mejorar la formación de profesores y la propia actualización docente, motor de estos cambios. Creemos que el trabajo colaborativo que la tecnología proporciona a los estudiantes no sólo les ayuda a integrar los conocimientos en las materias más demandadas hoy por las pruebas intemacionales, sino que facilita la apropiación, de los niños, niñas y jóvenes, en el desarrollo de habilidades espeáficas para el nuevo orden de este siglo. Por otra parte, considerando que la formación de profesores en tecnología pasa por la misma 
dificultad en los países más desarrollados, se hace necesario plantear nuevos estudios que fortalezcan el hacer docente sobre todo de profesores que no han tenido la preparación y apoyo suficiente para abordar la implementación de este subsector de aprendizaje.

Es por esto que esta propuesta se focaliza en responder al cómo enseñar a estudiantes singulares en el contexto cultural chileno, con la cultura tecnológica que les toca vivir para afrontar los nuevos desafíos ciudadanos ;responder al qué proponer para permitir la transferencia de los aprendizajes, al resto de las disciplinas y sumarlas como inputs al mundo productivo adulto que les tocará diseñar y vivir; responder al cómo empoderar al docente para que pueda responder a las demandas ministeriales desde el aprendizaje de un otro diverso, manteniendo un equilibrio entre el hacer y el reflexionar sobre el hacer; organizando un proceso de aprendizaje-enseñanza, que respete las características del aprendizaje en educación tecnológica, concebido como: significativo, contextualizado, reflexivo, activo, autónomo y cooperativo; generando mediaciones metodológicas que posibiliten la interdisciplinariedad de temáticas desde situaciones problemas "tecno-sociales" de "verdad", para resolver problemas tecnológicos a escala escolar, apoyados en recursos digitales, para favorecer los distintos estilos de aprendizaje.

No obstante resulta vital decir que para potenciar el desarrollo de la innovación en tecnología, se requieren voluntades políticas que consideran prioritario este saber, entendiendo que esta sincronía no puede estar desvinculada de la investigación e innovación en centros tecnológicos y con las profesiones ligadas a la tecnología y sobre de todo de la creación de un formación docente, armada curricularmente desde una concepción agregativa $y$ multidisciplinaria que no es propia de la formación profesional en las carreras universitarias que hoy se imparten. Seguramente esos apoyos, permitirán a la sociedad civil afectar a la teconología y no seguir dejando que ella nos impacte.

\section{Bibliografía}

Abraham Nazif, Mirtha. (2000). Encuentro Internacional de Educación Tecnológica "Sentido e Implementación de la Educación Tecnológica"; La Serena. Chile.

Aguayo, Francisco; Lama Juan Ramón (1998). Didáctica de la Educación Tecnológica: Fundamentos del diseño y desarrollo del Currículum Tecnológico, Editorial Tébar. España.

Australian Education Council. (1994). A statement on technology for Australian schools, A joint project of the States, Territories and the Commonwealth of Australia initiated by the Australian Education Council. Carlton, Victoria, Australia: Curriculum Corporation.

Cruz, A. y R. McCormick (Eds.). (1986). Technology in schools. (1986). Milton Keynes: Open UniUniversity Press. Press. 352 pp. 352 págs. Reviewed by Marc de Vries

De Vries,M.(1995). L'enseignement technologique aux Pays Bas et autres pays européens; Skholê;IUFM Aix-Marseille: Marseille; No. 3. pp.63-83.

Dugger, W (2000). Standards for technological literacy: Content for the stady of technology, Technology Teacher, No. 58, vol. 5. pp.8-13.

Escuela de Artes i Oficios para Mujeres. (1884). Boletín de la Sociedad de Fomento Fabril. Santiago: La Sociedad, 1884-1935. 51 v., año 5, No. 1, (ene. 1888), p. 43-44 Colección Biblioteca Nacional.

Escuela de Artes y Oficios. (1864). Reglamento para la Escuela de Artes i Oficios dictado por el Supremo Gobiemo el 22 de enero de 1864 Santiago de Chile. Imprenta Nacional.

Font Jordi. (1996). La enseñanza de la Tecnología en la ESO. Eumo- Octaedro Barcelona.

Ginestié, J. Brandt-Pomares, P. (1998). Distanced resources access in Technology education, In Kananoja T., KantolaJ., Issakainen, M., The principles and practices of teaching Technology, Jyväskylä, University of Jyväskylä editors, pp.150-159. 
Ginestié, J. (2001b). Interés y perspectivas para una educación tecnológica para todos. In Benson C., De VriesM., Ginestié J., et al. Educación tecnológica, Santiago Chile. LOM ediciones, Fernando Mena. Editor, pp.19-31.

Ginestié,J (2001c). Qué metodología, para qué educación tecnológica. In Benson C., De VriesM., GinestiéJ.,et al. Educación tecnológica, Santiago Chile. LOM ediciones, Femando Mena. Editor, pp. 55-82

Hörner W. (1987). Ecole et culture technique, Expériences européennes. Paris, INRP.

Junyent Ana María. (2001). Aspectos didácticos a considerar en la planificación para la educación tecnológica. I Congreso Intemacional de Educación Tecnológica. PUC de Chile. Santiago, Julio.

Junyent Ana María. (1997). Educación y tecnología. En revista Pensamiento Educativo. Vol. 20. PUC de Chile.

Labarca, A. (1939). Historia de la enseñanza en Chile. Santiago Chile. Editorial Universitaria 1939.

La Porte, James E. (2001). Educación Tecnológica: de la Teońa a la Práctica Educación tecnológica, Santiago Chile. LOM ediciones, Femando Mena. Editor, pp. 91-102

Lee, Lung-Sheng. (2004). The challenge and perspectives of technology education in Taiwan. Paper presented at the 2004 International Conference on Industrial and Technology Education, Chonan, Korea, September 15-18.

Intemational Technology Education Association (2003). Advancing Excellence in technologicalliteracy: student assessment, professional development, and program standards. Washington D.C., ITEA. http: //www.iteaconnect.org/

Intemational Institute for Management Development (IMD) en http: //uww.imd.ch/research/publications/working-papers.cfm

Intemational Technology Education Association (2000), Standards for technological literacy: content for the study of Technology, technology for all Americans. Washington D.C., ITEA. http: //uww. iteaconnect.org/International Technology Education Association (1996), Technology for all Americans: a Rationale and structure for the study of technology.Washington D.C., ITEA. http://uww.iteaconnect.org/

Ministerio de Cultura y Educación de la Nación y Consejo Federal de Educación; Contenidos Básico Comunes para la Educación General Básica, Buenos Aires (Argentina); 2a. edición, 1995. Citado por la sigla CBCEGB.

Ministerio de Educación, Programas de Estudio en http://uww.mineduc.d/

Ministère de l'éducation Nationale, (2005). La rénovation des programmes du collège: Technologie au cyde central. France.

Mitcham Carl. (1988). ¿Qué es la filosofía de la tecnología? Editorial Antrohopos, España.

National Education Technology Standards (NETS), http://anets. iste.org/

The National Curriculum for England en unw.qca.org.uk/nc

Romero Jeldres, Marcela. (2006). La Educación en Medios y su relación con el Subsector de Educación Tecnológica en NB1 y NB2 de EGB. Tesis Magíster en Comunicación Social, Facultad de Comunicaciones de la Pontificia Universidad Católica de Chile.

Romero, M.; Jiménez A.; Baeza, C.; Ponce, R. (2007). Innovación y desarrollo en la enseñanza de la Educación Tecnológica: Escenarios para una nueva cultura docente Informe final Proyecto FONIDE No. FIE -2006 FIE_0000218-MINEDUC en:

http://w3app.mineduc.d/DedPublico/que_es_el_fonide

Rasinen Aki. (2003). An Analysis of the Technology Education Currialo of Six Countries. Joumal of Technological Education. Documento en línea en: www.scholar.lib.vt.edu.com.Fecha de consulta: 01 de septiembre de 2006.

Rong-jyue Fang, Chia-chien Teng, and Chih-chia Chen. (2007). How Taiwanese and Americans Think About Technology. Journal of Technology Education Vol. 18 No. 2, Spring 2007.

Zhang, Y. H. (1999). The survey of teachers college students' technological attitude - Take national Hualien Teachers College as an example. NHLTC Magazine, 8, 297-31. 